\title{
NEURAL NETWORKS TO IDENTIFY PARTICLES USING TOPOLOGICAL PROPERTIES OF CALORIMETERS
}

\author{
Denis Oliveira Damazio* \\ Denis.oliveira.Damaziodcern.ch
}

\author{
José Manoel de Seixas* \\ seixaselps.ufrj.br
}

* Laboratório de Processamento de Sinais (LPS)

COPPE/EE/UFRJ, CP 68504, Rio de Janeiro 21945-970, Brazil

\begin{abstract}
The present work describes a neural particle classifier system based on topological mapping of the segmented information provided by a high-energy calorimeter, a detector that measures the energy of incoming particles. The achieved classification efficiencies are above $97.50 \%$ for the higher energy particle beams, even when experimental data exhibit unavoidable contamination due to the particle beam generation process, what could jeopardize the classifier performance. Some deterioration in the performance for the lower energy range is also discussed. The reduction on the dimensionality of the data input space caused by the topological mapping may be very helpful when online implementation of the classifier is required.
\end{abstract}

KEYWORDS: Neural networks, calorimeters, electronic instrumentation.

\section{RESUMO}

O presente trabalho apresenta um sistema classificador baseado em redes neurais aplicadas a um mapeamento topológico da informação segmentada fornecida por um calorimetro de altas energias. Este detector mede a energia das particulas incidentes em feixes experimentais. A eficiência de classificação atingida fica acima de $97,50 \%$ para feixes de mais alta energia, mesmo quando os dados experimentais apresentam uma inevitável contaminação ocasionada durante o processo

\footnotetext{
Artigo submetido em 12/12/02

1a. Revisão em 24/06/03

Aceito sob recomendação do Ed. Assoc. Prof. Cairo L. Nascimento
}

de produção das partículas. Alguma deterioração da qualidade do classificador encontrada para feixes de mais baixa energia também é discutida. A redução no número de dimensões do espaço de entrada, causada pelo mapeamento topológico, pode ser muito importante quando a implementação do classificador para operação online for considerada.

PALAVRAS-CHAVE: Redes neurais, calorímetros, instrumentação eletrônica.

\section{INTRODUCTION}

In the search for increasing the knowledge on the structure of matter, a bunch of collider experiments have been constructed and operated. To probe deeper into matter, CERN (The European Laboratory for Particle Physics, 2002) is now building a next generation particle accelerator. Such accelerator will use superconductivity to create huge magnetic fields which are necessary to maintain the particles on their tracks while an enormous amount of energy is transferred to them. From the particle collisions, it is expected to be found important reactions that may prove or disprove theoretic models in high-energy physics.

This new accelerator, LHC (Large Hadron Collider, 2002), will collide bunches of protons at periods of 25 nanoseconds and having $14 \mathrm{TeV}$ in the center of mass, when it starts to operate by the year 2007. LHC will have two main collision points, around each a complex set of detectors will be responsible for studying the products of the collisions. One of these particle detectors will be "A Toroidal LHC ApparatuS" (ATLAS Collaboration, 1994). This detector (see Fig. 1) will 


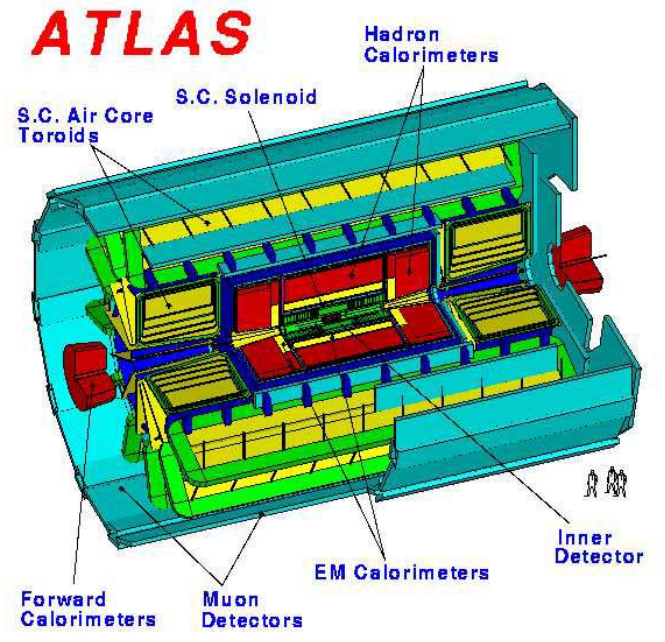

Figure 1: The ATLAS detector.

comprise tracking detectors (to display the trajectories of the particle coming from the impact point), calorimeters (to measure the energy of the particles) and other specific detectors, such as the muon chambers to detect muons, an important particle to the LHC physics.

One of the calorimeters of the ATLAS detector is Tilecal (F. Ariztizabal et al., 1990), which is specialized to detect the energy of hadronic particles, such as pions. This calorimeter is now in the calibration phase, in which calorimeter modules are submitted to particle beam tests. For detector calibration, beam selection comprises the type of particle to be injected (electrons, pions, and muons are used) and the energy (typically, energy selection ranges from $20 \mathrm{GeV}$ to $180 \mathrm{GeV}$ ). Despite the high quality achieved by the beam line nowadays, it is unavoidable particle contamination, when a specific beam selection is made. Thus, muons are present (up to 30\%) in pion beam selections and both muons and pions can be detected (up to $70 \%$ level of contamination has been observed) in electron beam selections. Such contamination levels tend to mask the actual performance of the calorimeter and affect the accuracy of the calibration process. Muon beams can be considered contamination free.

The purpose of this work is to use the calorimeter information for online rejection of particle contamination (outsider particles). As the calorimeter absorbs the energy of the incoming particles and it is segmented into a number of cells, it provides a detailed information of the energy deposition profiles, so that particle classes can be identified.

The online rejection of outsiders allows calibration data to be recorded free of contamination. For achieving high parti-

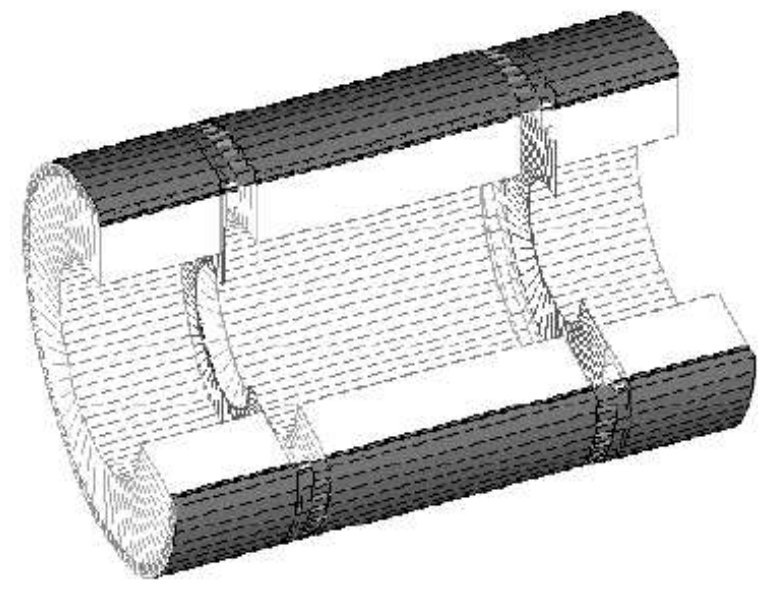

Figure 2: The structure of TileCal.

cle classification efficiencies and fast response, a topological mapping that groups calorimeter cells is developed. Such compacted calorimeter information is fed into the neural classifier for final particle identification, in terms of electrons, pions or muons.

The paper is organized into four sections. The next section will describe the calorimeter properties and the topological mapping used. The third section will focus on the results of the neural network application. Finally, the fourth section will derive some conclusions.

\section{HADRONIC CALORIMETER}

The hadronic calorimeter will form a toroid around the ATLAS collision point. The structure of TileCal can be seen in Fig. 2. It comprises a central barrel and two extended barrels, each one made of 64 modules. Products of the collisions will enter into TileCal at different impact points, defining regions of interest that will be analyzed in terms of energy and how the energy is deposited in the calorimeter.

The calorimeter is responsible for estimating with high accuracy the energy of the incoming particles. To be able to produce the energy measurement, the calorimeter is made of absorber plates (iron) sandwiched with plates of an active material (scintillating tiles). The incoming particles loose their energy by interacting with atoms of the absorber material. As a result of such interactions, many subproducts are generated and will also be traveling through the calorimeter mass, interacting with other atoms and, thus, creating a shower of particles with decreasing energy. The scintillating material is sensible to the energy produced by such shower of particles, so that light is produced proportional to the energy being sampled by the active material. This light is carried to 


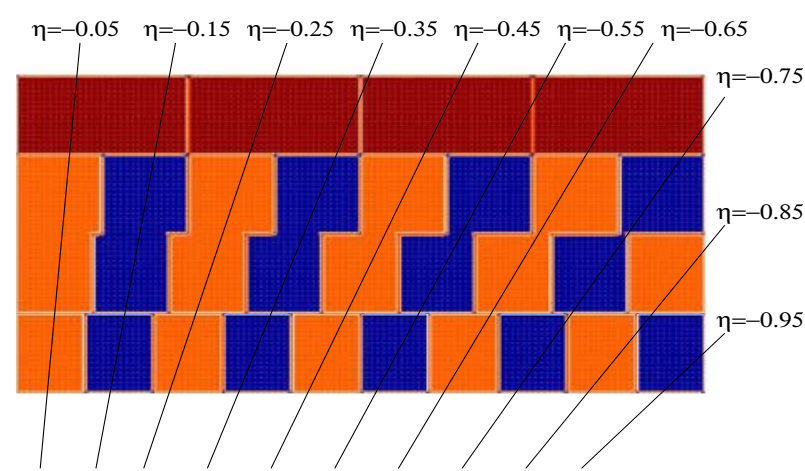

Figure 3: Cell topology in a calorimeter module and the trigger tower definition.

the outsider part of the detector by means of optical fibers, which are coupled to the tiles.

Detector segmentation into cells is produced by grouping fibers in the calorimeter modules and connecting them to photomultiplier tubes, which convert the light into electrical signals. The energy deposited in the detector can be estimated by adding the information readout from each calorimeter cell. Fig. 3 shows the cell topology.

As marked in Fig. 3, towers of cells are formed in the calorimeter structure, representing possible particle tracks in the calorimeter. These towers will be used for particle identification at LHC, in order to reduce the high event rate (40 $\mathrm{MHz}$ ) of LHC (ATLAS Level-1 Trigger Group, 1998). For this, an online triggering system is being designed and it relies strongly in the information obtained from the energy deposited in each tower of TileCal (Cerqueira et al., 2001). For the present work, 10 such trigger towers were formed by grouping the cells along the pseudorapidity directions (from $\eta=0.05$ up to $\eta 0.95$ ).

Each type of particle has a specific deposition profile in the calorimeter (Wigmans, 2000). For example, a typical electron event is displayed in Fig. 4, top. The heights in this figure represent the amount of energy deposited in each cell. As it can be inferred from this figure, electrons deposit almost all of its energy in the first cell they touch. On the other hand, typical pion profiles tend to achieve a deeper maximum, reaching the second layer of cells and spreading more energy around such maximum (Fig. 4, middle). However, higher fluctuations in pion profiles are observed, making pion events to come closer to the electron pattern.

Muons typically deposit just a small fraction of their energy in the calorimeter (Fig. 4, bottom), being easier to discriminate from the other two classes of particles, which deposit
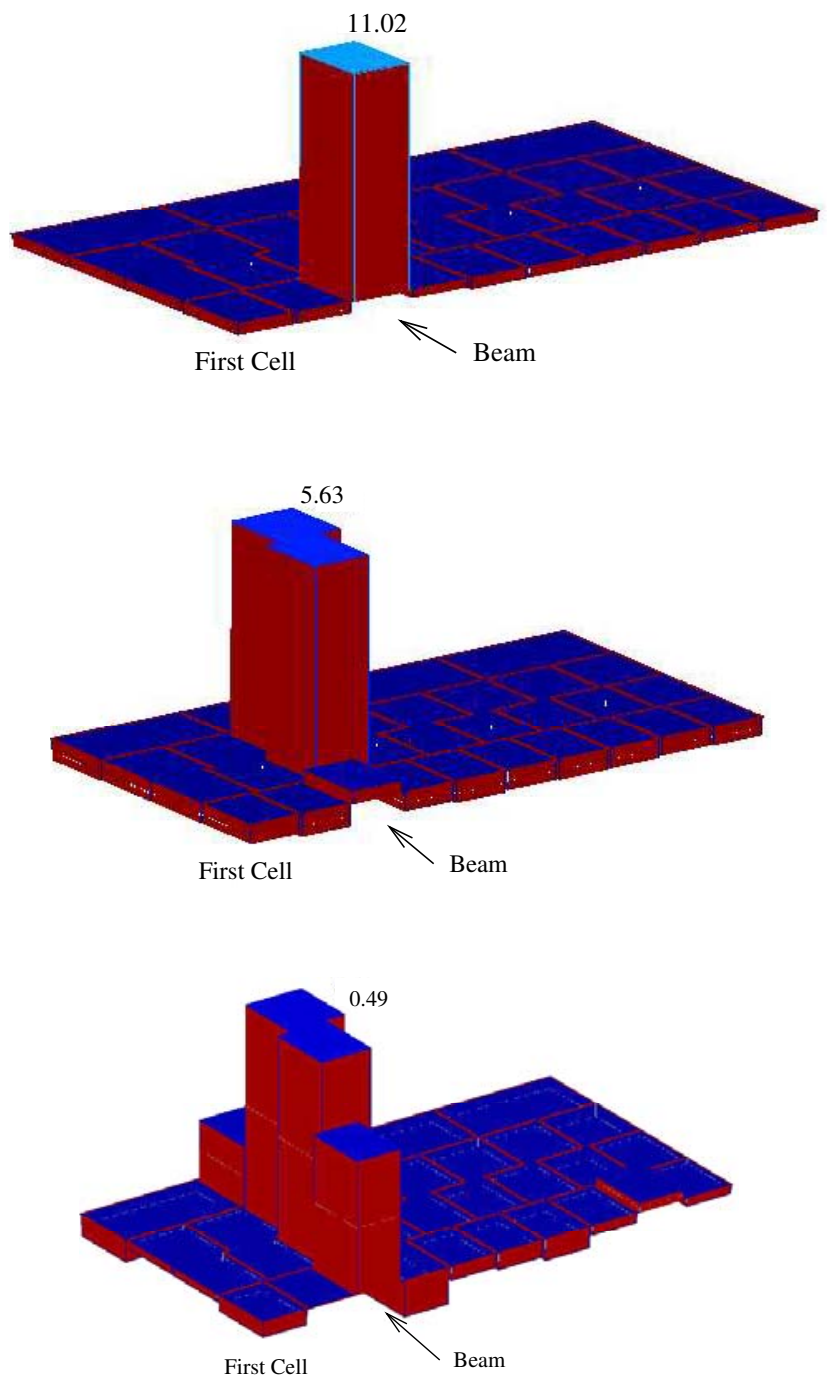

Figure 4: Typical electron (top), pion (middle) and muon (bottom) events.

around the nominal value of the beam ${ }^{1}$.

Discriminating between particles with calorimeter information is then a pattern recognition problem, for which neural networks (Haykin, 1999) may be an interesting choice. Feeding a neural classifier with such detailed energy deposition profiles (obtained from cell readout), a high performance particle identification system may be designed (Denby, 1999). However, as it was already mentioned, particle beams used for calibrating the calorimeter can be contaminated by outsider particles. This leads to a higher degree of difficulty in the classification task, since the pure data can only be ob-

\footnotetext{
${ }^{1}$ some energy leakage can be observed due to the restricted dimensions of the single module used in this work. For full calorimeter (192 modules, as in Fig. 2), energy leakage is negligible
} 
tained from a muon beam line.

The training method chosen for the neural classifier design was backpropagation, which is a supervised training method that requires the presentation of input-output pairs for adapting the neural network weights (Haykin, 1999). As a significant amount of events may be outsiders in a given data set acquired from a beam test, target values for the network training will be wrongly assigned. Despite such error in inputoutput assignment, it will be shown that the neural classifier can quickly recognize the energy deposition profile provided by the calorimeter for each particle class and, based on this, identify the actual class of the incoming particle. As online operation is envisaged, the network input information will be provided by the trigger tower mapping of the energy deposition profile. This data compaction allow to optimize the processing speed for both training and production phases of the network, so that the input data rate of the acquisition system will not be deteriorated by the neural processing.

\section{RESULTS}

For designing the neural network and evaluating its classification efficiency, the Jet Net-2.0 package (Lönnblad et al, 1992) was used. The multilayer neural network topology was optimized to satisfy both performance and compaction criteria. The final topology comprised 10 input nodes (to be fed by the ten trigger tower signals), 8 hidden nodes and 3 output nodes. Each output node was assigned to a given particle class (electrons, pions or muons).

Experimental data was composed of data sets related to each beam acquisition (muons, pions with contamination and electrons with contamination). Each data set contained more than 8000 events. These data sets were split into two sets, one used in the training phase of the network design and the other used to test the generalization power of the classifier. Due to the high level of statistics of the experimental data sample for all classes, training efficiencies were fully reproduced by the testing sets.

In spite of data contamination, for the training phase data acquired from a given beam selection were labeled according to the beam type, and only a single output was activated by the target vector. For instance, for electron beams of different energies $(20,100$, and $180 \mathrm{GeV})$, all suffering from both muon and pion contamination, acquired events were labeled as electrons, even though muons and pions should be present in the data sample. For this, the target values were +1 (activation) for the electron output node and -1 for the other output nodes. In the testing phase maximum probability was used to determine to which class the incoming particle would belong (Trees, 1971).
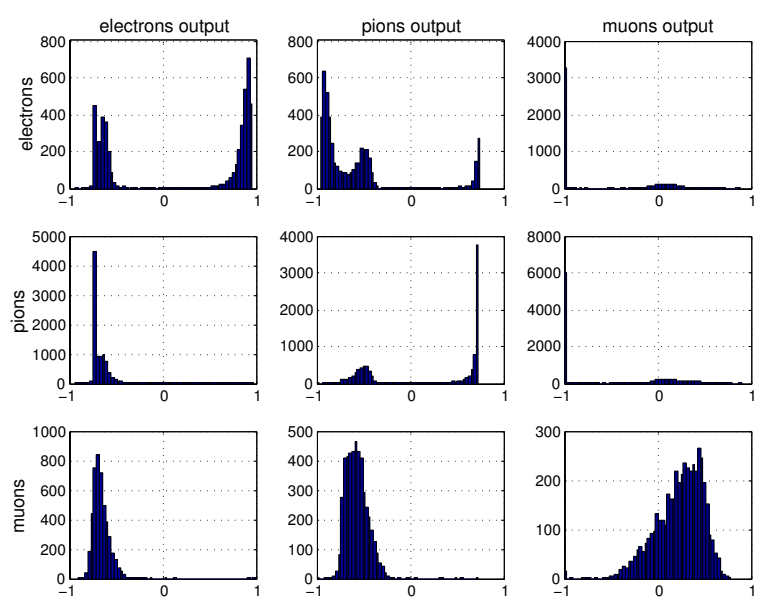

Figure 5: Neural network outputs (columns) for the three data sets (rows). See text

The hyperbolic tangent was the activation function selected for all neurons and the mean squared error, computed between the actual network output and the target vector for each event, was the figure of merit of the training phase. The stop criterium for the training phase was the maximum efficiency, which was computed by the product of the efficiencies over all particle classes.

For each event the trigger tower values were normalized by the square root of the absolute value of the total energy absorbed by the calorimeter (all trigger tower signals added up). This normalization scheme envisaged to retain some information on the level of the energy deposited by each incoming particle, which is quite relevant for discriminating electron and pions from muons (as from Fig 4, muons deposit very small amount of energy in the detector).

Fig. 5 shows a matrix of plots that exhibits the network response for the testing sets, at the end of the training phase (100 GeV case). Data coming from electron, pion and muon beam selections are shown in the rows of this matrix and columns refer to the network output nodes. Without any contamination, the histograms in the main diagonal should be peaking around +1 , indicating output nodes correctly activated by the respective particle class. On the other hand, histograms out of the main diagonal would be all peaking around -1 , as output nodes should be inactive to particle classes different to the ones they are assigned to. However, due to contamination in both electron and pion beam selections, this is not the case. Examining the electron output for data from the electron beam selection (top-left histogram), not only the expected peaking structure near +1 is observed, but also a second distribution close to -1 reveals that for these events the electron output is inactive, which means that 

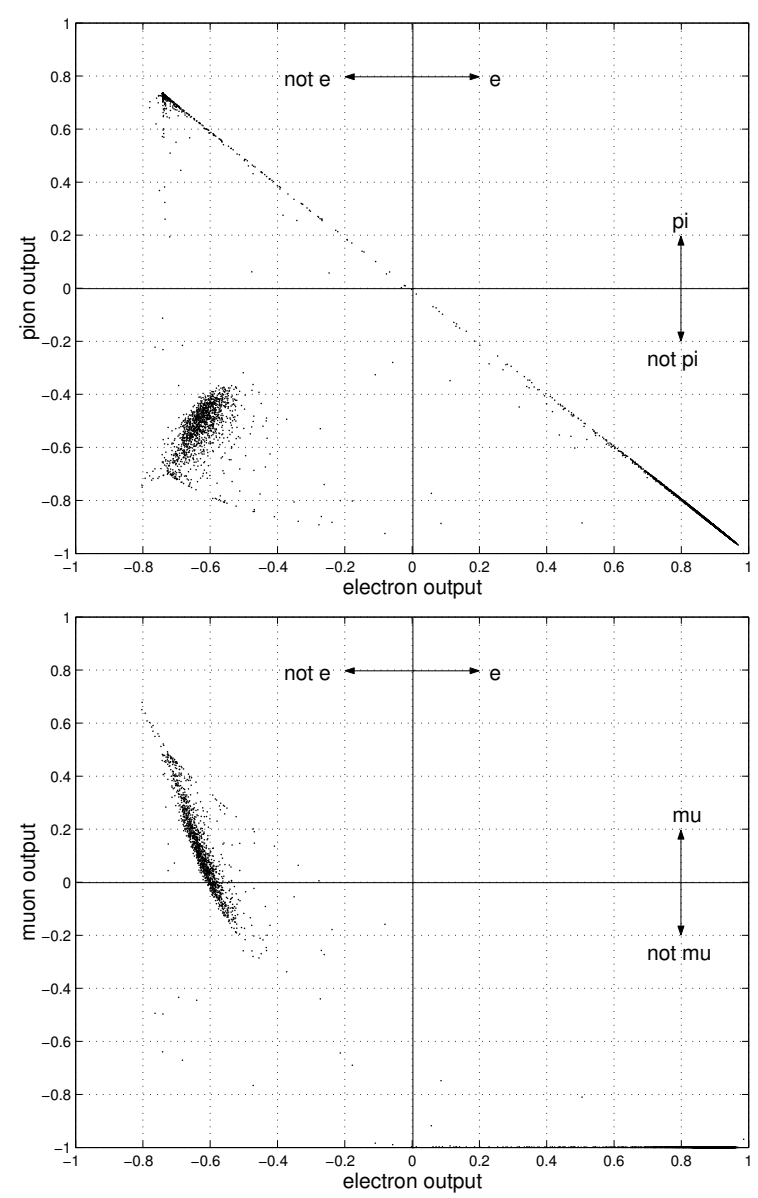

Figure 6: Correlation of the electron output and pion output (top). For the events with negative pion output, the correlation between the electron output and the muon output is also presented (bottom).

the electron output does not recognize them as electrons and thus declares them outsider particles in the data sample. The pion output (next histogram on the right) shows an agreement to the electron output, as it recognizes some events from the electron beam as pions by means of exhibiting two-peak structure, which includes a second peak near -1 .

To confirm this result, Fig. 6 presents the correlation between the electron output and the pion output (top) and th correlation between the electron and the muon outputs. As can be seen, the events considered by the electron output as being electrons (horizontal positive axis), were the same events considered not pions by the pion output (vertical negative axis). The events considered by the pion output as being pions, were the same events considered not electrons by the electron output. There are some events that received electron and pion output negative. The bottom figure shows the corre- lation between the electron and the muon output for the same events, except that the events with positive pion output were cut off. Again, the events considered to be electrons by the electron output were rejected as muons by the muons output and the events considered to be muons were rejected as electrons by the electron output. This means that the network shows to be able to identify incoming particles by their main features of energy deposition. Therefore, it can be concluded that the wrong labeling caused by beam contamination did not avoid the neural classifier to learn the detailed energy deposition profiles provided by the calorimeter.

Although the neural network response seems to understand the physics processes involved in the absorption of the incoming energy from different particle classes, validation of the particle identification is mandatory. As the application is intended to perform online rejection of outsiders, it should be guaranteed that the neural classifier does not introduce any bias in the data sample, allowing the offline analysis to be executed accurately.

The network response can be validated by means of exploitation of the main features of the shower development. As muons deposit a small fraction of their energy in the calorimeter, an energy cut suffices for muon identification, although detection efficiency decreases for lower energy beams. On the other hand, pions tend to travel much further in the calorimeter, with respect to electrons, so that the shower development in depth can be extremely powerful in electron/pion separation.

Considering data from pion beam $(100 \mathrm{GeV})$, Fig. 7 displays the correlation between the total energy and $O_{\pi}-O_{\mu}$, the subtraction of the muon output of the network from the pion output. As it can be seen, events in this plot with a positive $O_{\pi}-O_{\mu}$, which correspond to declared pions by the neural classifier, are those that deposited the largest energy in the detector. On the other hand, events with a negative $O_{\pi}-$ $O_{\mu}$ (so identified as muons by the network, as the electron output is not triggered by data from pion beam selections) are exactly those that deposited only a small fraction of their energy in the calorimeter. This agrees with the energy cut for separating muons from pions, which is typically established at $20 \mathrm{GeV}$ for $100 \mathrm{GeV}$ nominal beam energy.

Now, let's consider data from $100 \mathrm{GeV}$ electron beam. Outsider muon identification can be evaluated as above. For outsider pions, the detection is more difficult. Electrons and pions deposit basically the same amount of energy, but energy distribution in terms of calorimeter depth is different in general. To discriminate between electrons and pions, the fraction of the energy deposited in the first layer of cells can be used (as from Fig. 3, there are three layers of cells in depth). For instance, a cut at the level of $72 \%$ can be established, so 


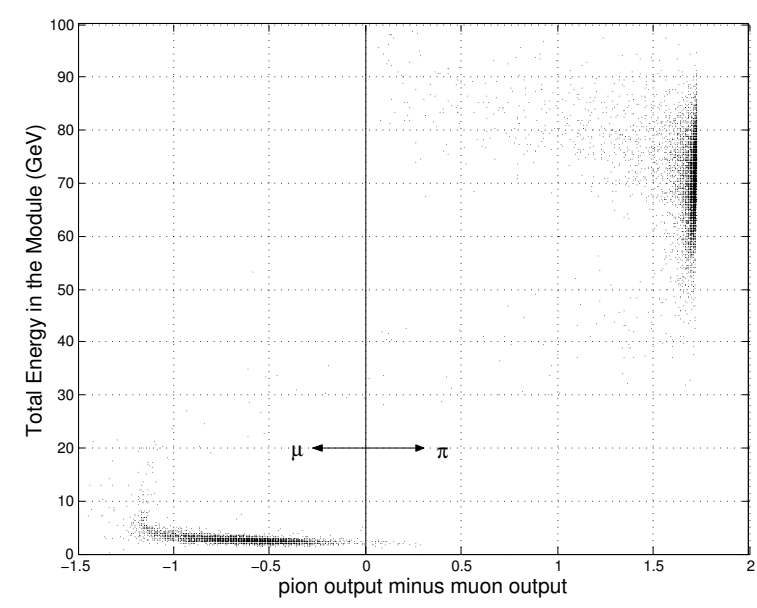

Figure 7: The subtraction of muon output from pion output correlated with the total energy deposited in the detector.

that events above this cut can be identified as electrons.

Figure 8 shows the correlation between the fraction of energy deposited in the first layer and $O_{e^{-}}-O_{\pi}$, the subtraction of the pion output from the electron output. This figure shows that the neural network response has also learned the energy deposition profile in depth, as most events that deposited more than $72 \%$ of their energy in the first layer had positive $O_{e^{-}}-O_{\pi}$, which means they have been declared as electrons by the neural classifier. In addition, most of events with negative $O_{e^{-}}-O_{\pi}$ (rejected electrons) deposited less than $72 \%$ of their energy in this first layer.

The expert information on the physics involved in shower development within the calorimeter can benchmark the neural processing, proving that the neural particle identification is unbiased. Classically, high-energy physicists combine detector information (total energy and shower development in depth) with data from auxiliary detectors (Cherenkov counters (Green, 2000) and especial muon detectors are typically used for improving outsider particle detection for lower energy values) for particle identification with calorimeters. This classical methodology can validate the neural classification (Seixas and Damazio, 1998), but it is quite hard to be implemented online, since it it is based on correlations that depend on beam. Also, classical methodology does not depend only on calorimeter information and for smaller energy beams it relies very much on auxiliary detector information, which increases the difficulties for online operation. As it will be shown next, neural processing works fine for different energy values and meets the requirement for online outsider rejection.

Table 1 shows the agreement between the classical method-

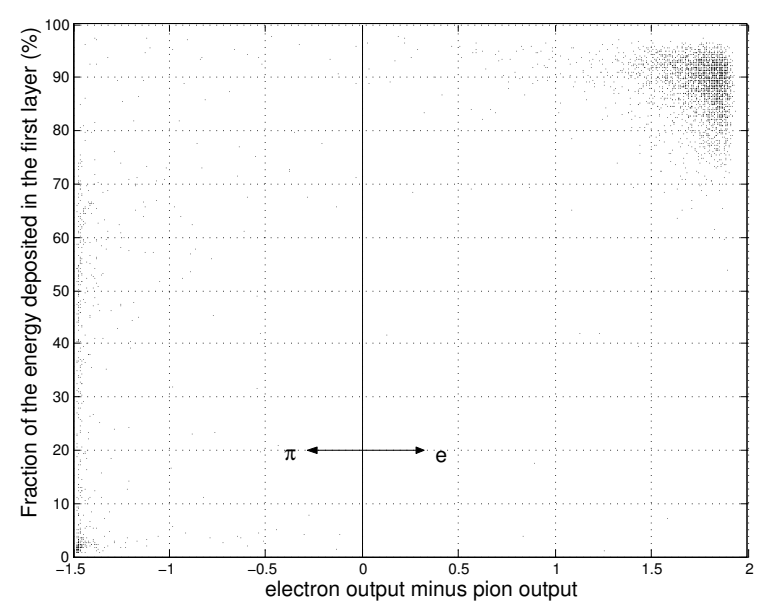

Figure 8: The subtraction of pion output from electron output correlated with the fraction of the energy deposited in the first detector layer.

Table 1: Agreement between neural and classical methodologies for $100 \mathrm{GeV}$ electron beam data.

\begin{tabular}{||c||c|c|c|}
\hline \multirow{2}{*}{\multicolumn{1}{|c|}{$\begin{array}{c}\text { Classical } \\
\text { Method }\end{array}$}} & \multicolumn{3}{c|}{ Neural Network } \\
\cline { 2 - 4 } & electron & pion & muon \\
\hline electron & $55.79 \%$ & $0.55 \%$ & $0.04 \%$ \\
\hline pion & $1.61 \%$ & $11.78 \%$ & $0.15 \%$ \\
\hline muon & $0.11 \%$ & $0.0 \%$ & $29.98 \%$ \\
\hline
\end{tabular}

ology and the neural network classification, considering 100 $\mathrm{GeV}$ electron beam. Total energy cuts for muon detection were placed at $20 \mathrm{GeV}$ and electron/pion separation used a cut in the energy deposited in the first layer at $72 \%$. In terms of contamination in the electron beam, both methods agree that pion contamination is at the level of $11.78 \%$ and outsider muons are present at a level of $29.98 \%$ of the data sample. Only $55.79 \%$ of the data sample was identified as electrons by both methods. The total agreement between the two techniques is $97.55 \%$, which is quite impressive.

Table 2 evaluates whether signal compaction by means of trigger towers deteriorates the particle identification efficiency. In this table, both neural classifier designs based on trigger towers and full detector granularity (46 cell energies) (Damazio and de Seixas, 1999) are compared for electron, pion and muon selections. Results are shown for overall agreement with classical methodology on particle detection for each $100 \mathrm{GeV}$ beam selection. As it can be inferred from this table, for electron and pion beams, signal compaction produced a gain in detection efficiency. On the contrary, for muons some deterioration in performance is observed. Averaging the efficiencies for the three beam selection, the com- 
pact classifier still performs better. In addition, for online application, the reduction of the complexity of the neural network, reducing the input nodes from 23 to 10 , is quite interesting as it can produce a faster classifier.

Table 2: Comparison between neural classifiers fed by trigger towers or full calorimeter granularity for $100 \mathrm{GeV}$ electron, pion and muon beams.

\begin{tabular}{||c||c|c|c|c|}
\hline agreem. & elec. & pion & muon & average. \\
\hline gran. & $95.30 \%$ & $94.37 \%$ & $99.50 \%$ & $96.39 \%$ \\
\hline tower & $97.55 \%$ & $97.62 \%$ & $97.80 \%$ & $97.66 \%$ \\
\hline
\end{tabular}

In order to check the dependency of the neural processing on beam energy, the neural classifier using trigger tower information was evaluated for other energy beam selections, covering low and high energy ranges. For both 100 and 180 $\mathrm{GeV}$ data, classical methodology employed the same cuts as referred to above. For $20 \mathrm{GeV}$ data, the total energy cut was set to $7 \mathrm{GeV}$ and the Cherenkov counter was used for performing electron/pion separation. The agreement between neural and classical methodologies is found in Table 3. Results are given in terms of overall agreement on each data sample, considering electron, pion and muon beams. As it can be depicted from this table, agreement is reduced for the $20 \mathrm{GeV}$ case, being more significant for pion beam selection. This can be explained by the fact that, at $20 \mathrm{GeV}$, the pion deposition profile is smaller in depth, being quite similar to the electrons' profile. In fact, $6.96 \%$ of the data sample, although recognized as pions by the classical methodology based on the Cherenkov counter, were identified as electrons by the neural network, which results in such disagreement.

Table 3: Agreement between neural and classical methodologies for different beam energies (in $\mathrm{GeV}$ ).

\begin{tabular}{||c||c|c|c|c|}
\hline Ener. & elec. & pion & muon & tot \\
\hline 20 & $92.68 \%$ & $89.69 \%$ & $96.98 \%$ & $93.12 \%$ \\
\hline 100 & $97.55 \%$ & $97.62 \%$ & $97.80 \%$ & $97.66 \%$ \\
\hline 180 & $97.70 \%$ & $94.42 \%$ & $99.48 \%$ & $97.20 \%$ \\
\hline
\end{tabular}

To further evaluate this shower development effect for 20 GeV, Fig. 9 displays the distribution for the sum of the energies deposited in the second and the fourth towers (the beam was entering into the calorimeter through the third layer, so these are the neighbor towers with respect to the impact point) for incoming particles from electron beam selections. The figure at the top shows the distribution for such sum of the trigger tower signals for $20 \mathrm{GeV}$ data and the one at the bottom the sum for $100 \mathrm{GeV}$. For $100 \mathrm{GeV}$, there is a relatively clear separation that can be established between electrons and muons (that only deposit energy in the main tower)
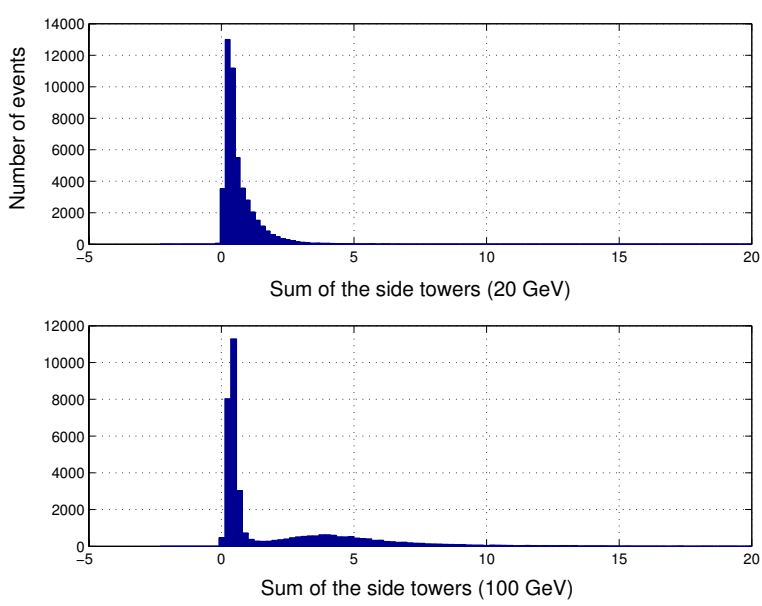

Figure 9: The sum of the towers around the main tower for 20 $\mathrm{GeV}$ (top) and $100 \mathrm{GeV}$ (bottom) electron beam selections.

and pions (that can leak energy to neighbor towers). For 20 $\mathrm{GeV}$, separation is much harder, as only a small energy tail is observed in the distribution. This means that, if the sum of the towers around the maximum is an important information for the neural discriminator, there is a clear explanation for the decrease in performance of the neural classifier for 20 $\mathrm{GeV}$ data.

So, it is important to evaluate which detector information is relevant to the neural classifier. To evaluate information relevance the method was the following: for each neural network trained (one for the $20 \mathrm{GeV}$ case and other to the 100 $\mathrm{GeV}$ case), the inputs (one at a time) were substituted by their mean value computed over the entire training set (including patterns from electron, pion, and muon beams with their inherent outsider particles). Then, the mean square error the outputs with relation to the outputs of the normal network is calculated for the entire set. In mathematical terms, the relevance of the input $j$ is :

$$
\text { relevance }(j)=\frac{1}{N} \Sigma_{i=1}^{N}\left|O u t_{\text {org }}(i)-O u t_{j}(i)\right|^{2}
$$

Where $O u t_{\text {org }}(i)$ is the output of the neural network for the original data set and $O u t_{j}(i)$ is the output of the same network on the set with the input $j$ changed by its mean. $N$ is the number of events on all data sets. If the input does not imply in a reasonably discrimination, the difference between its usage and the usage of the mean should not have an important impact on the neural network output. In the other way, if the input is important to the discrimination, then substituting it by its mean would produce a great error.

Fig. 10 presents the result of such analysis for the $20 \mathrm{GeV}$ case and $100 \mathrm{GeV}$ case. The first interesting point is that 

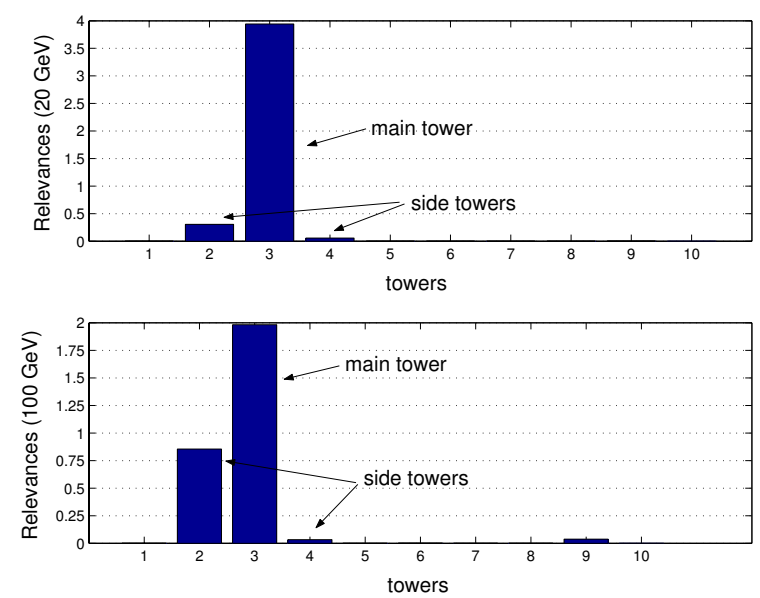

Figure 10: Relevances computed for each neural network input for the $20 \mathrm{GeV}$ case (top) and the $100 \mathrm{GeV}$ case (bottom).

the second tower has an information which is very similar to the fourth tower, since the particle energy spread can be imagined, in a simplified way, as a circle around the maximum (third tower), so touching equally the second and the fourth towers. This fact is recognized by the neural network that gives a relative importance to the second tower but treats the fourth one as redundant information by not assigning a very high relevance to it. We can observe that only the towers from 2 to 4 have a greater importance. This could lead to a procedure that uses just these towers as inputs to the network. Since our approach tried to keep the possibility of using the network for any tower, we decided to have all the towers. Also, as can be seen, a greater importance was assigned to the third layer at $20 \mathrm{GeV}$ case than at the $100 \mathrm{GeV}$ case, showing that most of the decision was taken based on that tower for $20 \mathrm{GeV}$, while for the $100 \mathrm{GeV}$ there is a relative greater importance given to the second tower. The relative importance of the second tower for $20 \mathrm{GeV}$ with relation to the maximum is more than 5 time smaller than the same measurement for $100 \mathrm{GeV}$.

This demonstrates that the neural network was capable to identify the smaller importance of the second (and fourth) tower, but, anyway the network was not capable to have a so high performance than in the higher energy cases. One possible solution is to usage of the fully calorimeter granularity, in order to observe the different energy deposition profiles inside a given tower. Using greater granularity at $20 \mathrm{GeV}$, it was possible to reach an agreement of $95.22 \%$ for the electron beam, $93.34 \%$ for the pion beam and $97.48 \%$ for the muon beam, $95.35 \%$ in the average, being comparable to the $100 \mathrm{GeV}$ case. The cost of this procedure is, as said before, a greater time for training and testing the neural network.

\section{CONCLUSIONS}

The neural methodology developed in this work employed a topological mapping based on trigger towers as a preprocessing method for a compact description of the information provided by a high-energy calorimeter. This design approach produced a high-efficiency particle discriminator that proved also to be able to identify outsider particles (contamination) in the data samples, even overcoming the wrong labeling of events in the supervised training of the classifier due to the existence of data contamination. This means that the backpropagation method could be used even when there is some inherent inconsistency in the data sample, which is an important result to the artificial intelligence field. Since it is unavoidable the wrong labeling of patterns, a design based on unsupervised methods, such as Kohonen networks, can probably be applied to particle discrimination with calorimeters. This shall be part of the developments of the future.

The results obtained in this paper have shown that a very good performance for the higher energy range could be achieved by the neural classifier, although some deterioration had been verified for the lower energy range $(20 \mathrm{GeV})$. This was explained by the fact that part of the relevant information was missing in the $20 \mathrm{GeV}$ case, because of the smaller energy spread in the calorimeter for $20 \mathrm{GeV}$ energy particles. The neural network proved to be able to identify such reduction in the relevance of some input towers information, but, due to the complexity of the problem, the network could not recover completely from this reduction in discrimination power of the calorimeter towers. In such case, the usage of the full calorimeter granularity could be a possible solution to profit from the tower internal energy distributions (Damazio et al., 2002). Another possibility would be to combine towers with the cells from the tower that had been hit by the incoming particle beam.

The developed methodology can be applied for online operation, profiting from the inherent input space dimensionality reduction and consequent increase in processing speed. This online classifier is envisaged to be used in the next beam test period of the calorimeter.

\section{ACKNOWLEDGMENTS}

We are thankful to the support given to this project by CAPES, CNPq, and FAPERJ (Brazil) and CERN (Switzerland). We would also like to thank our colleagues of the TileCal collaboration for fruitful discussions about this work and the data sets used. 


\section{REFERENCES}

ATLAS Collaboration (1994). Technical proposal for a general-purpose $\mathrm{pp}$ experiment at the large hadron collider at cern, Technical report, CERN. http://www.cern.ch/Atlas.

ATLAS Level-1 Trigger Group (1998). Level-1 technical design report, Technical report, CERN. http://atlas.web.cern.ch/Atlas/GROUPS/DAQTRIG/ TDR/tdr.html.

Cerqueira, A. S., de Seixas, J. M. and Caloba, L. P. (2001). An active adder for building the trigger signal of the hadronic calorimeter of atlas, IEEE Second SouthAmerican Workshop on Circuits and Systems.

Damazio, D. O. and de Seixas, J. M. (1999). Outsider identification in a neural particle discriminator based on calorimetry, Artificial Inteligence on High Energy Physics, Creta, Greece. http://www.lps.ufrj.br/ ${ }^{2}$ damazio/artigos/ aihenp299.ps.gz.

Damazio, D. O., Seixas, J. M. and Magacho, P. V. (2002). An online neural network triggering system for the tile calorimeter, IEEE Transactions on Nuclear Science 49(2): 369-376.

Denby, B. (1999). Neural networks in high-energy physics: a ten year perspective, Comp. Phys. Comm. 119(23): 219-231.

F. Ariztizabal et al. (1990). Construction and performance of an iron-scintillator hadron calorimeter with longitudinal tile configuration, A349: 384-397. http://atlasinfo.cern.ch/Atlas/ SUB_DETECTORS/TILE/tilecal.html.

Green, D. (2000). The Physics of Particle Detectors, Cambridge U. Press, New York, Estados Unidos. http://www.physicstoday.org/pt/vol-54/iss8/p47b.html.

Haykin, S. (1999). Neural Networks: A Comprehensive Foundation, Prentice-Hall.

Large Hadron Collider (2002). http://www.cern.ch/lhc.

Lönnblad et al (1992). Pattern recognition in high energy physics with artificial neural networks - jetnet 2.0, Comput. Phys. Commun. 70: 67.

Seixas, J. M. and Damazio, D. O. (1998). A neural discriminator capable to identify impurities in the data sample, IEEE International Conference on Electronics, Circuits and Systems - Lisbon, Portugal.
The European Laboratory for Particle Physics (2002). CERN. http://www.cern.ch.

Trees, H. V. (1971). Detection, Estimation and Modulation Theory, John Wiley \& Sons.

Wigmans, R. (2000). Calorimetry Energy Measurement in Particle Physics, Oxford. http://www.oupusa.org/isbn/0198502966.html. 